\section{An interval approach for stability analysis; Application to sailboat robotics}

Luc Jaulin, Fabrice Le Bars

OSM, Lab-STICC, ENSTA-Bretagne, 29806 Brest.

\begin{abstract}
This paper proposes an interval based method for the validation of reliable and robust navigation rules for mobile robots. The main idea is to show that for all feasible perturbations, there exists a safe subset of the state space such that the system cannot escape. The methodology is illustrated on the line following problem of a sailboat and then validated on an actual experiment where an actual sailboat robot, named Vaimos, sails autonomously from Brest to Douarnenez (more than $100 \mathrm{~km}$ ).
\end{abstract}

Index Terms-differential inclusion, interval analysis, line following, robotics, sailboat, stability, viability.

\section{INTRODUCTION}

Interval analysis [18] is an efficient tool for solving nonlinear problems. In the domain of robotics and automatic control, it has been used to study rigorously the stability of difficult linear [17] or nonlinear systems [26], to characterize capture domains [27][16], to compute nonlinear controllers [10] and to build reliable observers [21][7][1]. In this context, there exists also some point numerical techniques [23] which use some Lipschitz properties of the systems or ellipsoidal methods [22] when the system is linear. Now, interval methods can take advantage of constraint propagation tools to provide efficient resolution algorithms [5] and their ability to integrate nonlinear state equations in a guaranteed way [8]. When the system is both nonlinear and uncertain, in a set-membership context, stability analysis is a difficult problem and to our knowledge, no reliable algorithm are available in this context. The goal of the paper is twofold. It first shows that interval analysis can also be used for reliable stability analysis of uncertain nonlinear systems. Then, the paper deals with an actual autonomous uncertain system which is a sailboat robot. The principle of the approach is to represent uncertain systems by differential inclusions [2] and then to perform a Lyapunov analysis in order to transform the stability problem into a set-inversion framework. An illustration related to the validation of a control law for a sailboat robot [4][25] will be provided.

The paper is organized as follows. Section II presents the notion of $V$-stability which is derived from Lyapunov theory for stability analysis of nonlinear systems. It also shows that the $V$-stability can be cast into a set inversion problem which can be solved efficiently and in a guaranteed way by interval-based algorithms. Differential inclusions are a generalization of state equations when set-membership uncertainties occur. This notion is presented in Section III, and the $V$-stability is extended to deal with differential inclusions. It is also shown how interval based methods can be used to prove their $V$ stability. In Section IV, the approach is illustrated on the problem of finding a controller for a sailboat which is $V$-stable and which also satisfies some feasibility constraints. A convincing experimental validation demonstrates the applicability and the robustness of the resulting controller. Section V concludes the paper.

\section{II. $V$-STABILITY}

Consider a system described by the following state equation

$$
\dot{\mathbf{x}}=\mathbf{f}(\mathbf{x}) \text {. }
$$

This system may correspond to some controlled robot moving in an autonomous mode. Consider a differentiable function $V: \mathbb{R}^{n} \rightarrow \mathbb{R}$. The system (1) is said to be $V$-stable if there exists $\varepsilon>0$ such that

$$
(V(\mathbf{x}) \geq 0 \Rightarrow \dot{V}(\mathbf{x}) \leq-\varepsilon) \text {. }
$$

This definition, clearly influenced by the book of Aubin and Frankowska [2], has the main advantage that it can be studied using numerical methods. The notion of $V$-stability is weaker than the stability in the sense of Lyapunov [15]. Figure 1 illustrates a $V$ stable system which has a limit cycle (represented by the circle). Define the $V$-invariant set (painted gray in the picture) by $\mathbb{V}=$ $V^{-1}(]-\infty, 0[)=\{\mathbf{x} \mid V(\mathbf{x})<0\}$. Note that $\mathbb{V}$ is not necessarily bounded. The following theorem tells us that if the system is $V$-stable then it will be captured by $\mathbb{V}$.

Theorem 1. If (1) is $V$-stable then

$$
\begin{aligned}
& \text { (i) for all } \mathbf{x}(0), \exists t \geq 0 \text { such that } \mathbf{x}(t) \in \mathbb{V} \\
& \text { (ii) } \mathbf{x}(t) \in \mathbb{V} \Rightarrow \mathbf{x}([t, \infty[) \subset \mathbb{V} \text {. }
\end{aligned}
$$

Proof. Let us first prove (i). If $\mathbf{x}(0) \in \mathbb{V}$, the proposition is trivial. If now $\mathbf{x}(0) \notin \mathbb{V}$. Since $\dot{V}(\mathbf{x}(t)) \leq-\varepsilon$ as long as $V(\mathbf{x}(t)) \geq 0$, then $\exists t_{1} \in\left[0, \frac{V(\mathbf{x}(0))}{\varepsilon}\right]$, such that $V\left(\mathbf{x}\left(t_{1}\right)\right)=0$. The property (i) of (3) is thus satisfied. We shall prove (ii) by contradiction. Assume now $V(\mathbf{x}(t))<0$ and $V(\mathbf{x}(t+\tau)) \geq 0$ with $\tau>0$. Then $\exists t_{1} \in$ $[t, t+\tau]$ such that $V\left(\mathbf{x}\left(t_{1}\right)\right)=0$ and $\dot{V}\left(\mathbf{x}\left(t_{1}\right)\right) \geq 0$, which is inconsistent with (2).

Remark. Figure 2 gives an example where we have $(V(\mathbf{x}) \geq 0 \Rightarrow \dot{V}(\mathbf{x})<0)$ and (i) is not satisfied. This situation cannot appear if the property $(V(\mathbf{x}) \geq 0 \Rightarrow \dot{V}(\mathbf{x}) \leq \varepsilon<0)$ is true, as required by the definition of $V$-stability.

The following theorem shows that proving the $V$-stability amounts to solving a set inversion problem.

Theorem 2. Consider a small real number $\varepsilon>0$ and define the function

$$
g_{\varepsilon}(\mathbf{x})=\min \left(\frac{\partial V}{\partial \mathbf{x}}(\mathbf{x}) \cdot \mathbf{f}(\mathbf{x})+\varepsilon, V(\mathbf{x})\right) .
$$

We have the two following relations

$$
\begin{array}{lll}
\text { (a) } g_{\varepsilon}^{-1}([0, \infty[)=\emptyset & \Rightarrow & \text { (1) is } V \text {-stable } \\
\text { (b) } g_{0}^{-1}([0, \infty[) \neq \emptyset \Rightarrow & \text { (1) is } V \text {-unstable. }
\end{array}
$$

Proof. We shall first prove (a). Assume that for some given $\varepsilon$ we have $g_{\varepsilon}^{-1}\left(\left[0, \infty[)=\emptyset\right.\right.$, i.e., $\forall \mathbf{x}, g_{\varepsilon}(\mathbf{x})<0$. From (4), we get that for all $\mathbf{x}$, we have $\frac{\partial V}{\partial \mathbf{x}}(\mathbf{x}) . \mathbf{f}(\mathbf{x})+\varepsilon<0$ or

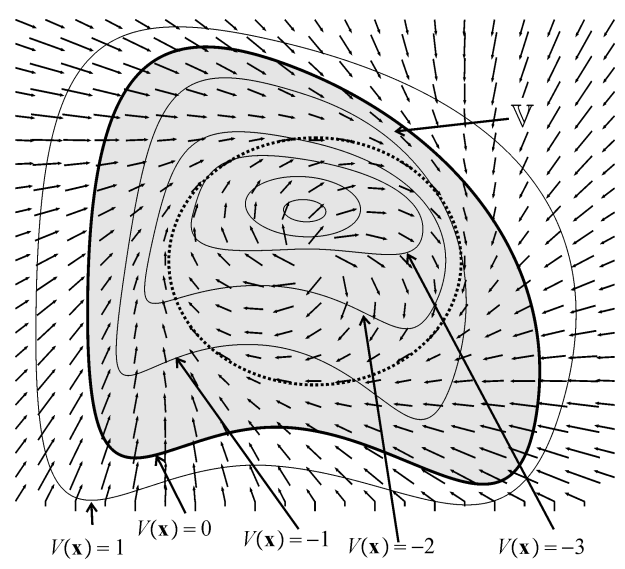

Fig. 1. If $V$ is the function represented by its level curves, then the system represented by the vector field is $V$-stable. 


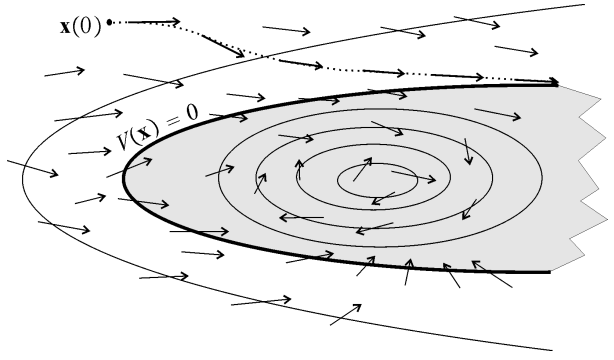

Fig. 2. Although $\dot{V}(\mathbf{x})<0$, the trajectory (dot line) never enters inside $\mathbb{V}$ (painted gray)

$V(\mathbf{x})<0$. Now, from the logic rule $(B$ or $\neg A) \Leftrightarrow(A \Rightarrow B)$, we get the following relation $\left(V(\mathbf{x}) \geq 0 \Rightarrow \frac{\partial V}{\partial \mathbf{x}}(\mathbf{x}) . \mathbf{f}(\mathbf{x})+\varepsilon<0\right)$. Or equivalently $(V(\mathbf{x}) \geq 0 \Rightarrow \dot{V}(\mathbf{x})<-\varepsilon)$. As a consequence, the system (1) is $V$-stable. The proof for (b) will be by contradiction. Assume that (1) is $V$-stable. Then from (2), we have the relation $(V(\mathbf{x}) \geq 0 \Rightarrow \dot{V}(\mathbf{x})<0)$ or equivalently $\left(V(\mathbf{x}) \geq 0 \Rightarrow \frac{\partial V}{\partial \mathbf{x}}(\mathbf{x}) . \mathbf{f}(\mathbf{x})<0\right)$. Thus, for all $\mathbf{x}$, we have $\frac{\partial V}{\partial \mathbf{x}}(\mathbf{x}) . \mathbf{f}(\mathbf{x})<0$ or $V(\mathbf{x})<0$, i.e., $g_{0}(\mathbf{x})<0$.

\section{DIFFERENTIAL INCLUSION}

When the system becomes uncertain, the state equation can be represented by a differential inclusion. This notion makes it possible to develop numerical algorithms to study rigorously the stability [23], [6]. This section presents the notion of differential inclusion and shows how the $V$-stability can be extended to uncertain systems.

\section{A. Thick functions}

A multivalued function (or thick function) $\mathbf{F}$ from $\mathbb{R}^{n} \rightarrow \mathbb{R}^{p}$ associates to each $\mathbf{x} \in \mathbb{R}^{n}$ a convex subset $\mathbf{F}(\mathbf{x})$ of $\mathbb{R}^{p}$ (see e.g., [3], [2]). Given a subset of $\mathbb{R}^{p}$, we define the lower and upper inverses [3] as follows

$$
\begin{aligned}
& \mathbf{F}^{-1}(\mathbb{Y})=\{\mathbf{x} \mid \mathbf{F}(\mathbf{x}) \subset \mathbb{Y}\} \\
& \overline{\mathbf{F}}^{-1}(\mathbb{Y})=\{\mathbf{x} \mid \mathbf{F}(\mathbf{x}) \cap \mathbb{Y} \neq \emptyset\} .
\end{aligned}
$$

Interval algorithms make it possible to compute efficiently guaranteed approximations of the upper and lower inverses by $\mathbf{F}$ of a set $\mathbb{Y} \subset \mathbb{R}^{p}$. Consider for instance the thick function $F\left(x_{1}, x_{2}\right)=$ $\left(x_{1}-[-1,1]\right)^{2}+\left(x_{2}-[-2,2]\right)^{2}=\left\{\left(x_{1}-a\right)^{2}+\left(x_{2}-b\right)^{2}, a \in\right.$ $[-1,1], b \in[-2,2]\}$. Interval arithmetic makes it possible to evaluate $F$. For instance $F(3,1)=(3-[-1,1])^{2}+(1-[-2,2])^{2}=$ $([2,4])^{2}+([-1,3])^{2}=[4,16]+[0,9]=[4,25]$. An accurate approximation of upper and lower inverses by $F$ of the interval $[10,100]$ is represented on Figure 3 . If $\mathbb{X}$ is the union of all black boxes and if $\overline{\mathbb{X}}$ is the union of black boxes with the two white rings then interval analysis guarantees the following enclosures $\mathbb{X} \subset$ $\underline{\mathbf{F}}^{-1}(\mathbb{Y}) \subset \overline{\mathbf{F}}^{-1}(\mathbb{Y}) \subset \overline{\mathbb{X}}$. Note that since the interval $[4,25]$ is neither inside nor outside $[10,100]$ the vector $(3,1)$ is inevitably inside the white zone.

\section{B. $V$-stability of differential inclusion}

Differential inclusions [2][20] are a generalization of the concept of state equation and are used to represent uncertain dynamic systems in a set-membership framework. A differential inclusion can be defined by the following inclusion

$$
\dot{\mathbf{x}} \in \mathbf{F}(\mathbf{x})
$$

where $\mathbf{F}$ is a thick function from $\mathbb{R}^{n}$ to $\mathbb{R}^{n}$. The differential inclusion (7) is said to be $V$-stable if all its solutions satisfy (2). It is said to be

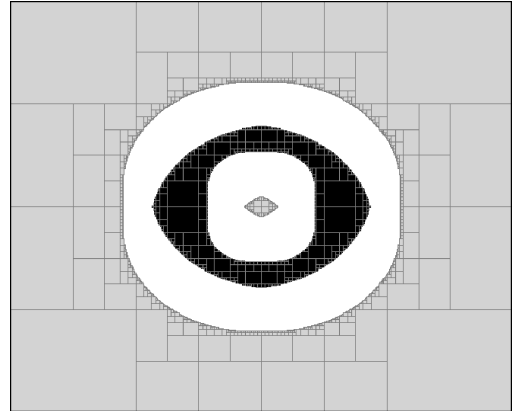

Fig. 3. Lower and upper set inversion of a thick function. The frame box corresponds to $[-20,20]^{2}$. Black boxes correspond to $\underline{\mathbf{F}}^{-1}(\mathbb{Y})$ and grey boxes do not intersect $\overline{\mathbf{F}}^{-1}(\mathbb{Y})$.

$V$-unstable if none of its solution satisfies (2). Since $\mathbf{F}(\mathbf{x})$ is closed and $V$ is differentiable, the thick function

$$
G(\mathbf{x})=\min \left(\frac{\partial V}{\partial \mathbf{x}}(\mathbf{x}) \cdot \mathbf{F}(\mathbf{x})+[\varepsilon], V(\mathbf{x})\right)
$$

where $[\varepsilon]=\left[0, \varepsilon^{+}\right]$is also an interval valued function. Here, the min operator should be understood in the Minkowski sense.

Theorem 3. If $G(\mathbf{x})$ is the thick function defined by (8), we have the two following relations

(a) $\bar{G}^{-1}([0, \infty[)=\emptyset \Rightarrow$ (7) is $V$-stable

(b) $\underline{G}^{-1}([0, \infty[) \neq \emptyset \Rightarrow$ (7) is $V$-unstable.

Proof. Let us first prove (a). $\bar{G}^{-1}([0, \infty[)=\emptyset \stackrel{(6)}{\Rightarrow}$ $\{\mathbf{x} \mid G(\mathbf{x}) \cap[0, \infty[\neq \emptyset\}=\emptyset \Rightarrow \forall \mathbf{x}, G(\mathbf{x}) \subset]-$ $\infty, 0\left[\stackrel{(8)}{\Rightarrow} \forall \mathbf{x}, \min \left(\frac{\partial V}{\partial \mathbf{x}}(\mathbf{x}) \cdot \mathbf{F}(\mathbf{x})+[\varepsilon], V(\mathbf{x})\right), \subset\right]-\infty, 0[\Rightarrow$ $\forall \mathbf{x}, \min \left(\frac{\partial V}{\partial \mathbf{x}}(\mathbf{x}) \cdot \dot{\mathbf{x}}+\varepsilon^{+}, V(\mathbf{x})\right)<0 \Rightarrow \forall \mathbf{x},\left(\dot{V}(\mathbf{x})<-\varepsilon^{+}\right.$or $V(\mathbf{x})<0) \Rightarrow\left(V(\mathbf{x}) \geq 0 \Rightarrow \dot{V}(\mathbf{x})<-\varepsilon^{+}\right) \Rightarrow$ (7) is $V$ stable. Let us now prove (b). $\underline{G}^{-1}([0, \infty[) \neq \emptyset \stackrel{(6)}{\Rightarrow}\{\mathbf{x} \mid G(\mathbf{x}) \subset$ $\left[0, \infty[\} \neq \emptyset \Rightarrow \exists \mathbf{x}, G(\mathbf{x}) \subset\left[0, \infty\left[\stackrel{(8)}{\Rightarrow} \exists \mathbf{x}, \min \left(\frac{\partial V}{\partial \mathbf{x}}(\mathbf{x}) \cdot \mathbf{F}(\mathbf{x})+\right.\right.\right.\right.$ $[\varepsilon], V(\mathbf{x})) \subset\left[0, \infty\left[\Rightarrow \exists \mathbf{x}, \min \left(\frac{\partial V}{\partial \mathbf{x}}(\mathbf{x}) \cdot \dot{\mathbf{x}}, V(\mathbf{x})\right) \geq 0 \Rightarrow\right.\right.$ $\exists \mathbf{x},(\dot{V}(\mathbf{x}) \geq 0$ and $V(\mathbf{x}) \geq 0) \Rightarrow(7)$ is $V$-unstable.

Consequence. Using a set inversion algorithm, we are able to prove that a differential inclusion is or not $V$-stable by computing two subpavings (i.e., union of boxes) $\underline{\mathbb{X}}, \overline{\mathbb{X}}$ such that $\underline{\mathbb{X}} \subset \underline{G}^{-1}([0, \infty[) \subset$ $\bar{G}^{-1}([0, \infty[) \subset \overline{\mathbb{X}}$. Taking (9) into account, we conclude that if $\underline{\mathbb{X}}$ is not empty, (7) is $V$-unstable and if $\overline{\mathbb{X}}$ is empty then (7) is $V$-stable.

Parametric case. Assume now that the robot depends on a parametric vector $\mathbf{p}$, i.e., it can be described by the following differential inclusion

$$
\dot{\mathbf{x}} \in \mathbf{F}(\mathbf{x}, \mathbf{p}) \text {. }
$$

The components of $\mathbf{p}$ correspond either to some tuning parameters that can be chosen arbitrary or to some perturbation vectors that cannot be chosen. We define the $V$-stability parameter set $\mathbb{P}$ as the set of all $\mathbf{p}$ such that the system is $V$-stable. Define the thick function $G_{\mathbf{p}}(\mathbf{x})=\min \left(\frac{\partial V}{\partial \mathbf{x}}(\mathbf{x}) \cdot \mathbf{F}(\mathbf{x}, \mathbf{p})+[\varepsilon], V(\mathbf{x})\right)=$ $\left\{\min \left(\frac{\partial V}{\partial \mathbf{x}}(\mathbf{x}) . \mathbf{f}+\varepsilon, V(\mathbf{x})\right), \mathbf{f} \in \mathbf{F}(\mathbf{x}, \mathbf{p}), \varepsilon \in[\varepsilon]\right\}$. From (9), we have (a) $\bar{G}_{\mathbf{p}}^{-1}\left(\left[0, \infty[)=\emptyset \Rightarrow \mathbf{p} \in \mathbb{P}\right.\right.$ and (b) $\underline{G}_{\mathbf{p}}^{-1}([0, \infty[) \neq \emptyset$ $\Rightarrow \mathbf{p} \notin \mathbb{P}$. As a consequence, if $\mathbb{P}^{-}=\left\{\mathbf{p}, \bar{G}_{\mathbf{p}}^{-1}([0, \infty[)=\emptyset\}\right.$ and $\mathbb{P}^{+}=\left\{\mathbf{p}, G_{\mathbf{p}}^{-1}\left([0, \infty[)=\emptyset\}\right.\right.$, then $\mathbb{P}^{-} \subset \mathbb{P} \subset \mathbb{P}^{+}$. Inner and outer subpavings [14], [24] approximations of $\mathbb{P}^{-}$and $\mathbb{P}^{+}$can be computed using interval analysis [13]. 


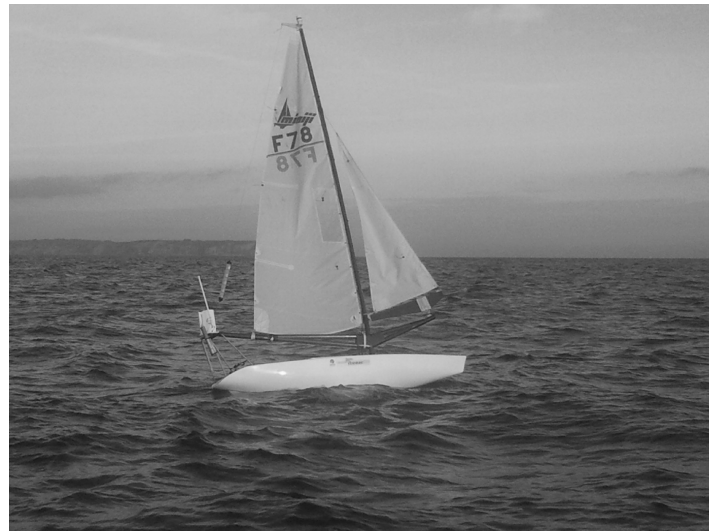

Fig. 4. The sailboat robot Vaimos

\section{APPLICATION TO SAILbOAT CONTROL}

\section{A. Controllers}

The robot to be considered here is the sailboat Vaimos of IFREMER (see Figure 4) which aims at collecting measurements at the surface of the ocean [9].

Heading controller. This robot has two inputs. One is the rudder angle $\delta_{r}$. We also control the maximal angle of the sail $\delta_{s}^{\max }$ (or equivalently, the length of the mainsheet). As a consequence, the actual angle of the sail satisfies $\delta_{s} \in\left[-\delta_{s}^{\max }, \delta_{s}^{\max }\right]$. Most of the time $\delta_{s}= \pm \delta_{s}^{\max }$ which means that the mainsheet is tight. Assume that the desired course angle $\bar{\theta}$ of the boat is feasible, then a simple control law for the rudder and the sail can efficiently make the robot move toward the right course $\bar{\theta}$. An efficient one is the heading controller [12] given by:

$$
\begin{cases}\delta_{r} & = \begin{cases}\delta_{r}^{\max } \cdot \sin (\theta-\bar{\theta}) & \text { if } \cos (\theta-\bar{\theta}) \geq 0 \\ \delta_{r}^{\max } \cdot \operatorname{sign}(\sin (\theta-\bar{\theta})) & \text { otherwise }\end{cases} \\ \delta_{s}^{\max } & =\frac{\pi}{2} \cdot\left(\frac{\cos (\psi-\bar{\theta})+1}{2}\right)\end{cases}
$$

where $\theta$ is the heading of the robot (measured by a compass) and $\psi$ is the angle of the wind measured by a weather vane. In (11), $\delta_{r}^{\max }$ is the maximal angle of the rudder and $\sin (\theta-\bar{\theta})$ corresponds to the heading error (we did not take $e=\theta-\bar{\theta}$ as an error: when $\theta-\bar{\theta}=2 k \pi$, we want a zero error). When $\cos (\theta-\bar{\theta})<0$, the robot is going to the opposite direction and the rudder is tuned at the maximum $\left(\delta_{r}= \pm \delta_{r}^{\max }\right)$. For the sail, we took a Cardioid model: when we are going downwind $\cos (\psi-\bar{\theta})=1$, the sail should be opened $\left(\delta_{s}^{\max }=\frac{\pi}{2}\right)$; when we are close to the wind $\cos (\psi-\bar{\theta}) \simeq$ -1 , the sail should be closed $\left(\delta_{s}^{\max } \simeq 0\right)$. Once the low level control loop has been implemented, a supervisor should send to the heading controller, feasible courses in order to perform the line following (see Figure 5).

Feasibility of a course. A course $\bar{\theta}$ is feasible if

$$
\cos (\psi-\bar{\theta})+\cos \zeta \geq 0,
$$

where $\zeta$ is the close hauled angle (typically $45^{\circ}$ ). If this condition is not satisfied, the course $\bar{\theta}$ corresponds to a direction which is too close to the wind and the boat cannot keep the course $\bar{\theta}$ (see Figure 6).

Vector field controller. Following a vector field has been proposed by [19] for sailboat robots. A vector field controller has the form $\bar{\theta}=h(\mathbf{x}, \psi)$, where $\mathbf{x} \in \mathbb{R}^{2}$ represents the position of the center of the robot (measured using the GPS). The algorithm below proposes the function $\bar{\theta}$, obtained using a pragmatic approach, that has been

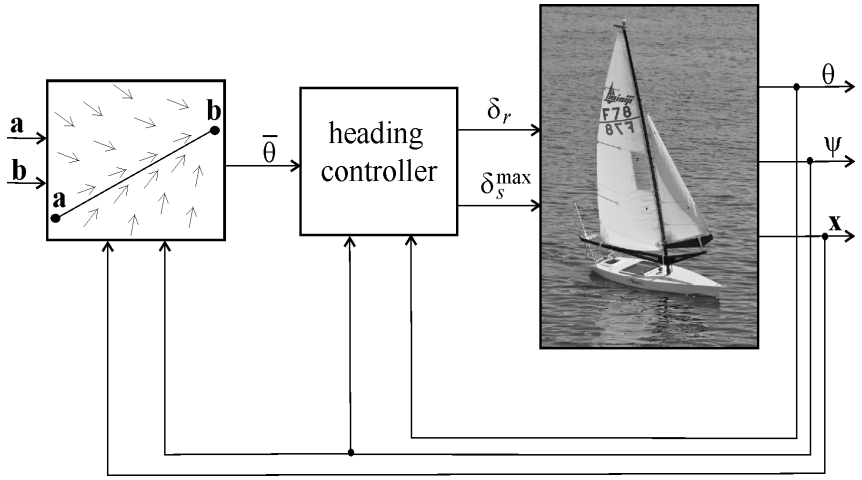

Fig. 5. The robot is controlled first by a loop to fix the heading and then by a second vector field loop to generate feasible headings

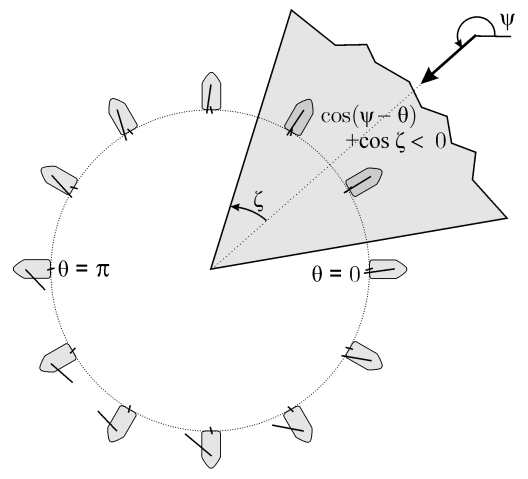

Fig. 6. Some directions for the sailboat are not feasible. These unfeasible courses forms the no-go zone painted grey.

implemented in Vaimos. The points $\mathbf{a}, \mathbf{b}$ correspond to the line to be followed.

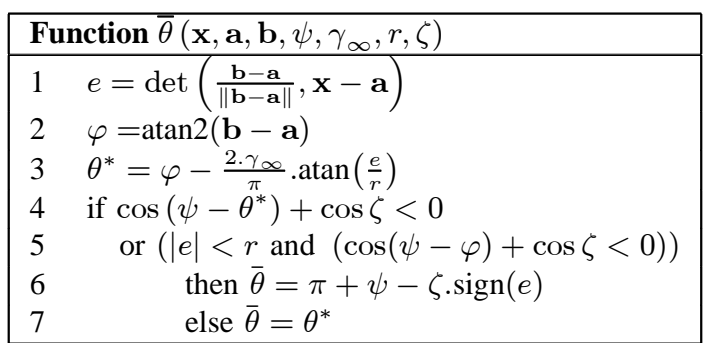

At Step 1, we compute the algebraic distance $e$ from the robot to its line $(\mathbf{a b})$. The sign of $e$ determines if the robot is on the left or on the right to the line, as represented by Figure 7. At Step 2, the angle of the line $\varphi$ is computed. Two modes should be taken into account: the direct mode and the close hauled mode. (1) Direct mode. This mode is chosen when the wind is well oriented so that tack manoeuvres are not needed. The corresponding nominal course $\theta^{*}$ (see Figure 7) is computed at Step 3 where $\gamma_{\infty}>0$ corresponds to the incidence angle (when the robot is far from its line, $\gamma_{\infty}$ corresponds to the angle between the desired heading and the line) and $r$ corresponds to the cutoff distance. This expression for $\theta^{*}$ makes the line attractive: (a) when $e= \pm \infty$, we have $\theta^{*}=\varphi \pm \gamma_{\infty}$, i.e., the robot has a heading which corresponds to the angle $\gamma_{\infty}$. (b) if $e= \pm r$, we have $\theta^{*}=\varphi \pm \frac{\gamma_{\infty}}{2}$ and (c) for $e=0$ we have $\theta^{*}=\varphi$, which corresponds to the direction of the line. (2) Close hauled mode. Two tacks can be chosen: the starboard tack or the port tack. We chose the right tack at Step 6. Now, it remains to choose the mode (direct or close hauled). If $\theta^{*}$ is not a feasible course (see (12)), then the close hauled mode 


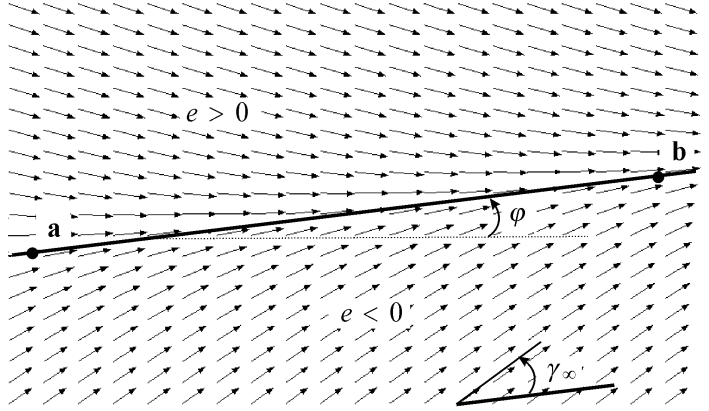

Fig. 7. Nominal vector field $\theta^{*}$ associated with the line following. The coefficient $\gamma_{\infty}$ allows to weight between two objectives: (i) the heading should corresponds to the direction of the line and (ii) the distance to the line should be small.

is chosen. It is also the case if the direction $\varphi$ of the line does not correspond to a feasible course, except if we are far from the line, i.e., $|e|<r$ (see Step 5).

\section{B. Characterization of the feasible parameter set}

We choose a frame based on the line to be followed. The origin of the frame is $\mathbf{a}$, and on this frame, $\varphi=0$. The function $\bar{\theta}$ becomes:

$$
\begin{array}{|lc|}
\hline \multicolumn{2}{|c|}{\text { Function } \bar{\theta}\left(\mathbf{x}, \psi, \gamma_{\infty}, r, \zeta\right)} \\
1 & \theta^{*}=-\frac{2 \cdot \gamma_{\infty}}{\pi} \cdot \operatorname{atan}\left(\frac{x_{2}}{r}\right) \\
2 & \text { if } \cos \left(\psi-\theta^{*}\right)+\cos \zeta<0 \\
3 & \text { or }\left(\left|x_{2}\right|<r \text { and }(\cos \psi+\cos \zeta<0)\right) \\
4 & \text { then } \bar{\theta}=\pi+\psi-\zeta \cdot \operatorname{sign}\left(x_{2}\right) ; \\
5 & \text { else } \bar{\theta}=\theta^{*} ; \\
\hline
\end{array}
$$

We assume that the heading controller generates an actual heading of $\theta \in\left[\bar{\theta}-e_{\theta}, \bar{\theta}+e_{\theta}\right]$. Moreover, we also suppose that the speed $v$ is always strictly positive. As a consequence, since the wind angle $\psi$ is inside the interval $[\psi]=[-\pi, \pi]$, studying the stability of the robot amounts to studying the stability of the differential inclusion

$$
\dot{\mathbf{x}} \in\left(\begin{array}{c}
\cos \left(\bar{\theta}\left(\mathbf{x},[\psi], \gamma_{\infty}, r, \zeta\right)+\left[-e_{\theta}, e_{\theta}\right]\right) \\
\sin \left(\bar{\theta}\left(\mathbf{x},[\psi], \gamma_{\infty}, r, \zeta\right)+\left[-e_{\theta}, e_{\theta}\right]\right)
\end{array}\right)
$$

This is due to the fact that the stability properties of nonlinear systems are invariant to any time transformation of the form $d \tau=v(t) . d t$, where $v(t)>0$. The properties of interest are the following. Property 1. If the robot has a distance to the line less than $r_{\max }=$ $50 \mathrm{~m}$ then, it will be the case forever. Property 2. If the robot has a distance greater than $r_{\max }=50 \mathrm{~m}$ then this distance will decrease until it reaches a distance less than $r_{\max }$. Property 3. The robot always moves toward the right direction, i.e., $\dot{x}_{1}>0$. The $V$-stable parameter $\mathbb{P}$ set corresponds to the set of all $\mathbf{p}$ such that Properties 1 and 2 are satisfied.

\section{Testcases}

The parameter vector is taken as $\mathbf{p}=\left(\gamma_{\infty}, \psi\right)$, where $\psi \in[-\pi, \pi]$ is the angle of the wind with respect to the line and $\gamma_{\infty} \in[0, \pi]$ is the incidence angle. Let us illustrate the principle of our approach on two testcases related to the line following of a sailboat robot. For both cases, we take $V(\mathbf{x})=x_{2}^{2}-r_{\max }^{2}$. Thus, the $V$-invariant set $\mathbb{V}$ corresponds to the strip centered onto the line with radius $r_{\max }$.

Case 1. We only take into account Properties 1 and 2. For $\left[-e_{\theta}, e_{\theta}\right]=[0,0],[\varepsilon]=\left[0,10^{-4}\right], \zeta=\frac{\pi}{3}, r=25 m$, the $V$-stable parameter set $\mathbb{P}$ is represented on Figure 8 . This characterization for $\mathbb{P}$ is obtained in less than 1 minute on a classical laptop. As illustrated by the white circle for $\gamma_{\infty}=\frac{\pi}{2}$, there exist some wind directions (for

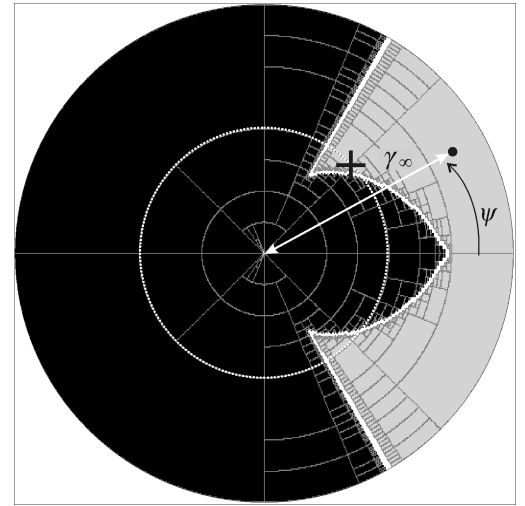

Fig. 8. Characterization of the $V$-stable parameter set $\mathbb{P}$ for Case 1 . The black area is proved to be inside $\mathbb{P}$ and the gray area is proved to be outside $\mathbb{P}$. The cross + represents a parameter vector for which the system is not $V$-stable.

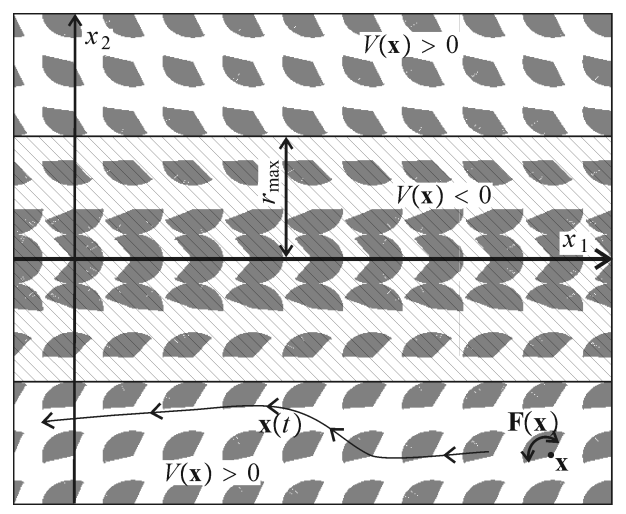

Fig. 9. Differential inclusion associated with the controlled sailboat for $\zeta=\frac{\pi}{3}$ and $\gamma_{\infty}=\frac{\pi}{2}$. The frame box is $[-100 \mathrm{~m}, 100 \mathrm{~m}]^{2}$.

instance $\psi=\frac{\pi}{4}$, as represented by the cross + ) for which the system is $V$-unstable. The differential inclusion associated to the white circle (i.e., $\gamma_{\infty}=\frac{\pi}{2}$ and $\psi \in[-\pi, \pi]$ ) is represented on Figure 9. The $V$ invariant set $\mathbb{V}=\left\{x_{2}^{2}-r_{\max }^{2}<0\right\}$ corresponds to the hatched strip. To draw this figure, we built a grid in the state space $\mathbf{x}=\left(x_{1}, x_{2}\right)$ and for each $\mathbf{x}$ we have drawn arrows corresponding to all $\dot{\mathbf{x}}$ consistent with the relation (13). The instability for some points of the white circle (such as the cross) can be interpreted by the existence of trajectories that are consistent with the differential inclusion and that do not satisfy Property 2 . Such an unstable trajectory $\mathbf{x}(t)$ is drawn on Figure 9.

Case 2. We also want $\dot{x}_{1}>0$ which corresponds to Property 3. Moreover, we take $e_{\theta}=5^{\circ}=0.085 \mathrm{rad}, r=25 \mathrm{~m}$ and $\zeta=\frac{\pi}{6}$. Figure 10 gives a characterization of the resulting $\mathbb{P}$ in less than 2 minutes. As illustrated by the white circle for $\gamma_{\infty}=\frac{\pi}{8}$, for all feasible perturbations and for all wind directions, the robot is always $V$-stable and all other constraints (feasibility of $\bar{\theta}$ and $\dot{x}_{1}>0$ ) are satisfied. This feasibility of the differential inclusion is illustrated by Figure 11.

\section{Experiment}

Figure 12 gives a track of $100 \mathrm{~km}$ that has been done autonomously from Brest to Douarnenez (Brittany, France) by the sailboat robot Vaimos. For the parameters of the controller, we took $r=25 \mathrm{~m}$, $\gamma_{\infty}=\frac{\pi}{4}$ and we checked that the resulting controller guarantees the $V$-stability, provided that Vaimos with its heading controller satisfy 


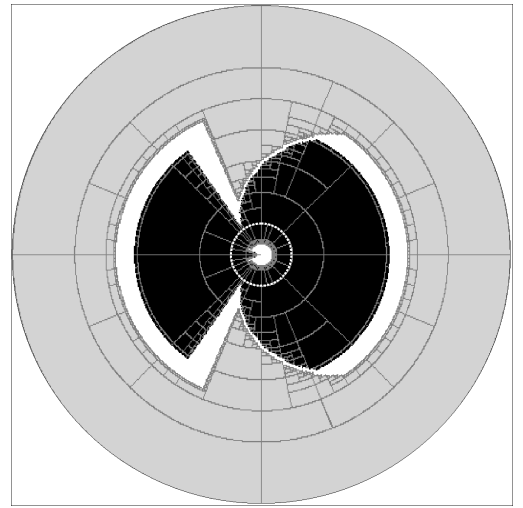

Fig. 10. $\quad V$-stable parameter set $\mathbb{P}$ for Case 2. The radius $\gamma_{\infty}$ of the white circle guarantees the $V$-stability for all wind.

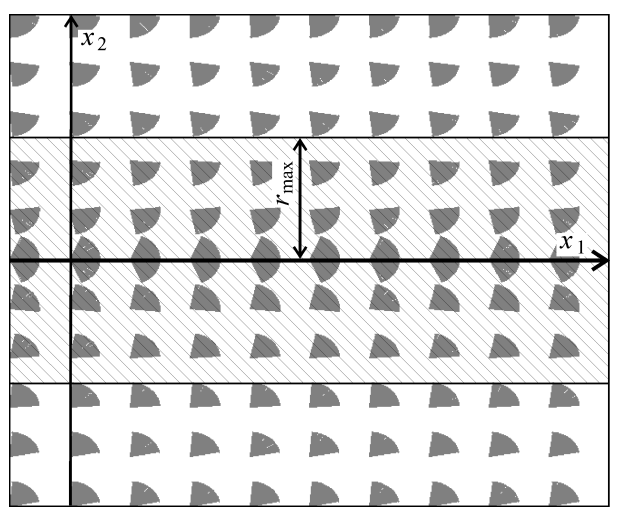

Fig. 11. Differential inclusion associated with the controlled sailboat for $\zeta=\frac{\pi}{6},\left[e_{\theta}\right]= \pm 0.085$ and $\gamma_{\infty}=\frac{\pi}{8}$.

(13). The wind comes from the south. Except when the robot was inside the circle (due to a submarine coming back to Brest, we had the duty to move the robot toward the south) and inside the triangle (to avoid a collision with a boat), the robot was always at a distance less than 30 meters to its line (where we proved that this distance should be smaller than $r_{\max }=50 \mathrm{~m}$ ). The properties $1,2,3$ are thus always satisfied, as expected. Since $\zeta$ is taken as $\frac{\pi}{3}$, it was not possible to satisfy Property 3 , which has been violated several times during the mission. Inside the square, the robot had to move upwind. It was in a close hauled mode and alternated starboard tacks with port tacks. More details related to this mission and to the method (photos, $\mathrm{C}++$ source code, videos) are available in [11].

\section{CONCLUSION}

This paper has proposed a new interval analysis approach to validate that a nonlinear uncertain robot will always satisfy some required properties, provided that normal working conditions are fulfilled. Of course in case of breakdown, atypical events, collisions, ... the state equations or the error bounds are not valid and nothing can be guaranteed anymore. This validation step is necessary if we want to build swarm of robots or to make the robot moving with humans where rules have to be followed and where responsibilities have to be pointed out in case of accident. The principle of the approach that has been proposed for the validation is to represent the robot with all perturbations by a differential inclusion. A Lyapunov methodology is then used to cast the stability requirements into a set inversion framework. All other feasibility constraints are then aggregated and the resulting problem becomes a set inversion problem involving

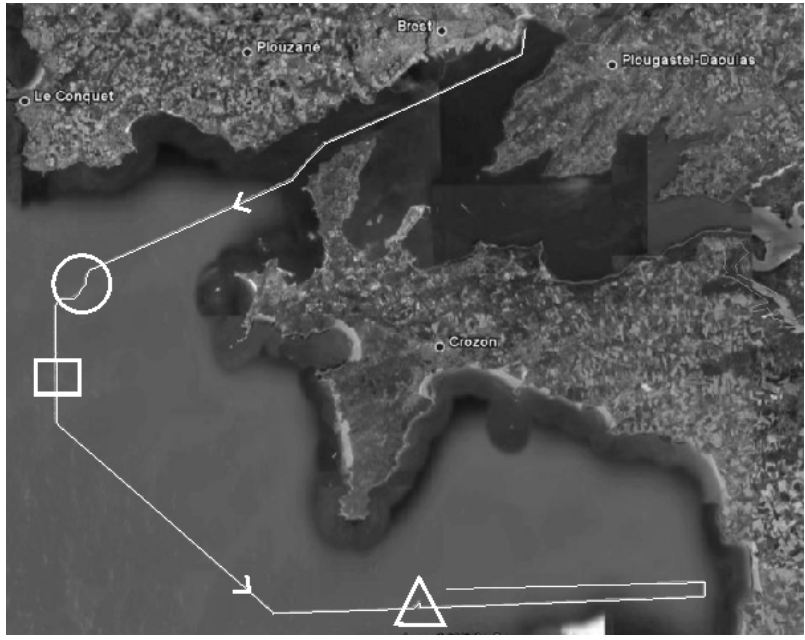

Fig. 12. Track of Vaimos during its trip from Brest to Douarnenez. The wind comes from the south.

multivalued functions (or thick functions). Now, this set inversion can efficiently be solved using interval analysis. The methodology has been illustrated on the control problem of an actual sailboat robot for which a large scale mission of more than $100 \mathrm{~km}$ has been performed. During its mission, the robot has never been at a distance more than 30 meters to its line. To our knowledge such an accurate track for a sailboat robot in the ocean has never been done before.

Acknowledgement. The robot Vaimos is the result of a collaboration between LPO (Laboratoire de Physique des Océans), RDT (Recherches et Développements Technologiques) of IFREMER (Institut Français de Recherche pour l'Exploitation de la Mer) and ENSTA-Bretagne (Ecole Nationale Supérieure de Techniques Avancées). The authors render thanks to LPO and RDT for having made available Vaimos to test the controllers presented in this paper. They also thank all people involved in the project: Y. Auffret, S. Barbot, L. Dussud, B. Forest, E Menut, S. Prigent, L. Quemeneur, P. Rousseaux (RDT, IFREMER); F. Gaillard, T. Gorgues, O. Ménage, J. Moranges, T. Terre (LPO) and B. Clément, Y. Gallou, O. Reynet, J. Sliwka and B. Zerr (ENSTA-Bretagne).

\section{REFERENCES}

[1] F. Abdallah, A. Gning, And P. Bonnifait. Box particle filtering for nonlinear state estimation using interval analysis. Automatica 44(3), 807-815 (2008).

[2] J. Aubin and H. Frankowska. "Set-Valued Analysis". Birkhäuser, Boston (1990).

[3] C. BergE. "Topological Spaces: Including a Treatment of Multi-Valued Functions, Vector Spaces and Convexity". Dover Books on Mathematics (1963).

[4] Y. BRIÈRE. "The first microtransat challenge, http: //web.ensica.fr/microtransat". ENSICA (2006).

[5] G. Chabert AND L. JAUlin. Contractor Programming. Artificial Intelligence 173, 1079-1100 (2009).

[6] E. Cruck, R. Moitie, AND N. Seube. Estimation of basins of attraction for uncertain systems with affine and lipschitz dynamics. Dynamics and Control 11(3), 211-227 (2001).

[7] V. DRevelle And P. Bonnifait. High integrity gnss location zone characterization using interval analysis. In "ION GNSS" (2009).

[8] A. Goldsztejn, W. Hayes, And P. Collins. Tinkerbell is chaotic. SIAM Journal on Applied Dynamical Systems 10(4), 1480-1501 (2011).

[9] T. Gorgues, O. Ménage, T. Terre, and F. Gaillard. An innovative approach of the surface layer sampling. Journal des Sciences Halieutique et Aquatique 4, 105-109 (2011).

[10] P. Herrero, L. Jaulin, J. Vehi, And M. A. Sainz. Guaranteed set-point computation with application to the control of a sailboat. 
International Journal of Control Automation and Systems 8(1), 1-7 (2010).

[11] L. JAULIN. "Programs and demo for sailboat controllers, available at www.ensta-bretagne.fr/jaulin/checking.html". ENSTA-Bretagne (2012).

[12] L. Jaulin, F. L. Bars, B. Clément, Y. Gallou, O. Ménage, O. REYNET, J. SLIWKA, AND B. ZERR. Suivi de route pour un robot voilier. In "CIFA 2012", Grenoble (France) (2012).

[13] L. JAULIN AND E. WALTER. Guaranteed tuning, with application to robust control and motion planning. Automatica 32(8), 1217-1221 (1996).

[14] L. Jaulin, E. Walter, O. LÉvêQue, And D. Meizel. Set inversion for $\chi$-algorithms, with application to guaranteed robot localization. Mathematics and Computers in Simulation 52(3-4), 197-210 (2000).

[15] H. KhaliL. "Nonlinear Systems, Third Edition". Prentice Hall (2002).

[16] M. Lhommeau, L. Jaulin, And L. Hardouin. Capture basin approximation using interval analysis. International Journal of Adaptative Control and Signal Processing 25(3), 264-272 (2011)

[17] R. Malti, X. Moreau, F. Khemane, and A. Oustaloup. Stability and resonance conditions of elementary fractional transfer functions. Automatica 47(11), 2462-2467 (2011).

[18] R. E. MOORE. "Methods and Applications of Interval Analysis". SIAM, Philadelphia, PA (1979).

[19] C. Petres, M. R. Ramirez, and F. Plumet. Reactive path planning for autonomous sailboat. In "IEEE International Conference on Advanced Robotics", pp. 1-6 (2011).

[20] M. QUINCAMPOIX. Differential inclusions and target problems. SIAM journal on control and optimization 30(2), 324-335 (1992).

[21] T. RAissi, N. RAMdAni, AND Y. CANDAU. Set membership state and parameter estimation for systems described by nonlinear differential equations. Automatica 40, 1771-1777 (2004).

[22] D. RokityansKi AND S. Veres. Application of ellipsoidal estimation to satellite control. Mathematical and Computer Modelling of Dynamical Systems 11(2), 239-249 (2005).

[23] P. SAINT-PIERRE. Approximation of the viability kernel. Applied Mathematics ans Optimization 29(2) (1994).

[24] R. SAINUdin. "Machine Interval Experiments: Accounting for the Physical Limits on Empirical and Numerical Resolutions". LAP Academic Publishers, Köln, Germany (2010).

[25] C. SAUZE AND M. NEAL. An autonomous sailing robot for ocean observation. In "proceedings of TAROS 2006", pp. 190-197, Guildford, UK (2006).

[26] W. TUCKER. The Lorenz attractor exists. Comptes Rendus de l'académie des Sciences 328(12), 1197-1202 (1999).

[27] J. WAN. "Computationally reliable approaches of contractive model predictive control for discrete-time systems". PhD dissertation, Universitat de Girona, Girona, Spain (2007). 